\title{
Mud-trapped herd captures evidence of distinctive dinosaur sociality
}

David J. Varricchio, Paul C. Sereno, Zhao Xi-jin, Tan Lin Jeffrey A. Wilson, and Gabrielle H. Lyon Acta Palaeontologica Polonica 53 (4), 2008: 567-578 doi:http://dx.doi.org/10.4202/app.2008.0402

A unique dinosaur assemblage from the Cretaceous beds of western Inner Mongolia preserves geologic and paleontologic data that clearly delineate both the timing and mechanism of death. Over twenty individuals of the ornithomimid Sinornithomimus dongi perished while trapped in the mud of a drying lake or pond, the proximity and alignment of the mired skeletons indicating a catastrophic mass mortality of a social group. Histologic examination reveals the group to consist entirely of immature individuals between one and seven years of age, with no hatchlings or mature individuals. The Sinornithomimus locality supports the interpretation of other, more taphonomically ambiguous assemblages of immature dinosaurs as reflective of juvenile sociality. Adults of various nonavian dinosaurs are known to have engaged in prolonged nesting and post hatching parental care, a life history strategy that implies juveniles spent considerable time away from reproductively active adults. Herding of juveniles, here documented in a Cretaceous ornithomimid, may have been a common life history strategy among nonavian dinosaurs reflecting their oviparity, extensive parental care, and multi-year maturation.

Key words: Dinosauria, Ornithomimosauria, taphonomy, herding, sociality, miring, drought, Cretaceous.

David J. Varricchio [djv@montana.edu], Earth Sciences, Montana State

University, Bozeman, MT 59717, USA; Paul C. Sereno [dinosaur@uchicago.edu], Department of Organismal Biology and Anatomy, 1027 E. 57th Street, University of Chicago, Chicago, IL 60637, USA; Zhao Xi-jin [zhaolu54@ 263.net], Institute of Vertebrate Paleontology and Paleoanthropology, Chinese Academy of Sciences, Beijing 100044, PRC; Tan Lin [firsttan@ sina.com], Long Hao Geologic and Paleontological Research Center, Department of Land Resources, Hohhot, Nei Mongol 010010, PRC; Jeffrey A. Wilson [wilsonja@umich.edu], Museum of Paleontology and Department of Geological Sciences, University of Michigan, Ann Arbor, MI 48109, USA; Gabrielle H. Lyon [glyon@ projectexploration.org], Project Exploration, 950 East 61st Street, Chicago, IL 60637, USA. 
This is an open-access article distributed under the terms of the Creative Commons

Attribution License (for details please see creativecommons.org), which permits unrestricted use, distribution, and reproduction in any medium, provided the original author and source are credited.

FaF 